\title{
Gender Minority Stress: A Critical Review
}

\section{Kyle K.H. Tan ${ }^{1}$, Gareth J. Treharne ${ }^{2}$, Sonja J. Ellis ${ }^{3}$, Johanna M. Schmidt ${ }^{4}$, Jaimie F. Veale $^{1}$}

${ }^{1}$ School of Psychology, University of Waikato, New Zealand

${ }^{2}$ Department of Psychology, University of Otago, New Zealand

${ }^{3}$ School of Human Development and Counselling, University of Waikato, New Zealand

${ }^{4}$ School of Social Science, University of Waikato, New Zealand

Author Note

Kyle K.H. Tan, Bsc (Hons), PhD Candidate

Gareth J. Treharne, $\mathrm{PhD}$

Sonja J. Ellis, PhD

Johanna M. Schmidt, PhD

Jaimie F. Veale, PhD

Correspondence concerning this paper should be addressed to Kyle K.H. Tan, School of Psychology, University of Waikato, Private Bag 3105, Hamilton 3240, New Zealand. E-mail: kht5@students.waikato.ac.nz 


\begin{abstract}
Past studies that compare cisgender to transgender (or trans) and gender diverse people have found a higher prevalence of mental health problems among the latter groups. This paper utilises Testa's Gender Minority Stress Framework, which is an expansion of Minority Stress Theory to assess minority stressors that are specific to the experiences of trans and gender diverse people. The concept of cisnormativity, an ideology that positions cisgender identities as a norm, is used in relation to the Gender Minority Stress Framework to describe the marginalising nature of social environments for trans and gender diverse people. This paper provides a critical review that integrates and expands upon past theoretical perspectives on gender minority stressors and protective factors. Specifically, this paper demonstrates the relevance of cultural and ethnic backgrounds to complement the application of intersectionality in research on health disparities experienced by trans and gender diverse people.
\end{abstract}

Keywords: transgender, gender diverse, gender minority stress, cisnormativity, intersectionality 


\section{Introduction}

Transgender, or the shorthand trans, is an umbrella term referring to people whose gender does not correspond with the sex assigned to them at birth, such as trans women and trans men (American Psychological Association, 2015). Gender diverse is a term that describes people whose gender identity or expression does not conform to societal expectations of gender in a binary construct (Adams et al., 2017). Gender diverse people may define their gender outside the binary constructs of "man" or "woman", identifying as neither men nor women (e.g., agender or non-binary); or as moving between binary genders (e.g., genderfluid) (American Psychological Association, 2015). Gender diverse people do not necessarily ascribe to a trans identity, particularly those of non-Western cultural backgrounds who might identify with non-Western terms that are best understood within their specific cultural context (e.g., Samoan fa'afafine and Cook Islands akava'ine) (Adams et al., 2017; Alexeyeff \& Besnier, 2014). In this paper, the abbreviation TGD is used to refer to trans and gender diverse to encompass all whose gender identity might differ from normative binary constructions of gender. Cisgender is used to refer to those whose gender identity aligns with their assigned physical sex in socially accepted ways (American Psychological Association, 2015).

This paper provides an integration and expansion of theories of mental health disparities relating to gender identity by (1) critically reviewing Meyer's conceptualisation of minority stress and its relevance to the experiences of TGD people; (2) engaging in an indepth reading of other explanatory frameworks, in order to discuss the effectiveness of Testa's (2015) GMSF in encapsulating gender-minority stressors and protective factors experienced by TGD people; and (3) utilising intersectionality to explain how TGD people with multiple marginalised identities may experience specific forms of minority stress. 
A number of review studies have investigated the prevalence of mental health problems among TGD people (see Dhejne, Van Vlerken, Heylens, \& Arcelus, 2016; McNeil, Ellis, \& Eccles, 2017; Millet, Longworth, \& Arcelus, 2017). These review studies found that TGD people manifest higher rates of various mental health problems when compared to either cisgender people or general population prevalence figures. For example, a systematic review of the prevalence of anxiety symptoms and disorders among TGD people across countries in the regions of Americas, Europe and Asia, found that the prevalence of anxiety among TGD people may be as high as $68.0 \%$, compared to the $18.0 \%$ found in general population surveys (Millet et al., 2017).

The high prevalence of mental health problems among TGD people has been found to correlate with the impact of minority stress. Minority stress is the "excess stress to which individuals from stigmatised social categories are exposed as a result of their social, often a minority, position” (Meyer, 2003, p. 675). Minority Stress Theory (MST) was originally developed by Meyer $(1995 ; 2003)$ to provide theoretical explanations for the effect of minority stress on the mental health of specific sexual minority groups, namely lesbian, gay and bisexual (LGB) people. Tokenistic incorporation of TGD people in the over-arching concept of sexual orientation and gender minorities (conventionally known as LGBT people) may seem to render MST applicable to TGD people. However, research demonstrates that the stressors experienced by TGD people on the basis of gender identity and expression are not necessarily the same as those experienced by LGB people (see Clarke, Ellis, Peel, \& Riggs, 2010). Testa and colleagues subsequently developed the Gender Minority Stress Framework (GMSF) (Testa, Habarth, Peta, Balsam, \& Bockting, 2015), which considers specific minority stressors that are unique to TGD people to elucidate the disparities in mental health problems between TGD and cisgender people.

\section{Gender Minority Stress}


The Gender Minority Stress Framework (Testa et al., 2015) is built on Meyer's Minority Stress Theory (Meyer, 1993; 2003). The GMSF employs a stress discourse to examine the social environments affecting TGD people (Testa et al., 2015). MST conceptualises stress based on the application of engineering analogy, in which stress is assessed as "a load relative to a supportive surface" (Meyer, 2003, p. 675). In this instance, a load is depicted as a stressor, which can be conceptualised as a stimulus that threatens the optimal functioning of a person's cognitive perception (Koolhaas et al., 2011). When a stressor becomes uncontrollable and unpredictable for an individual, the person concerned is said to be experiencing stress (Koolhaas et al., 2011). Meyer (2003) further postulated that loads can be differentiated into individual and social stressors and that people of minority groups are subjected to an additional form of social stressor that is not experienced by those in dominant groups- minority stress.

Riggs and Treharne (2017) argued that Meyer's definition of minority stress as a distinctive or additional form of social stress does not correspond with the understanding of the engineering analogy. Figure 1 depicts the difference in the application of engineering analogy between Meyer (2003) and Riggs and Treharne (2017). Riggs and Treharne (2017) raised an example to consider stressful social environments as a load which places social exertion on a supportive surface (i.e., a minority person or group). In this instance, the load has incorporated both individual and social characteristics of a stressor, thus generating a cumulative form of stress that exert its influences on a minority person. Given that stress is a product of cumulative effects of stressors, Riggs and Treharne (2017) refuted Meyer's view that minority stress arises from a person's marginalised social position mediated entirely via intra-individual factors such as internalised homophobia. Riggs and Treharne (2017) instead argue that minority stress should be perceived to originate from institutionalised ideologies and social norms that accord a minority position. Hence, in this review we conceptualise 
gender minority stress as a form of stress that is unique to TGD people, and that the minority position of TGD people is the product of marginalising ideologies and social norms that privilege cisgender people.

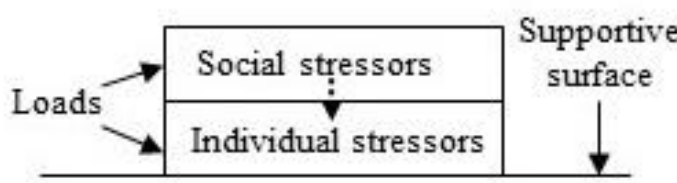

Meyer's (2003) perspective

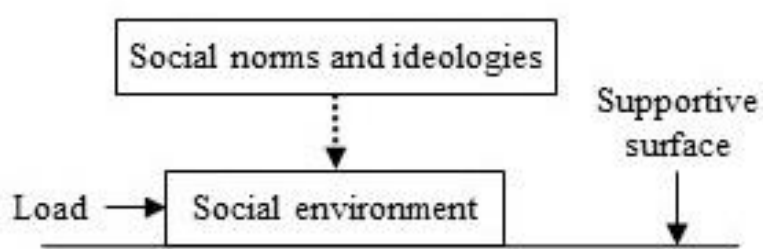

Riggs and Treharne's (2017) perspective

Figure 1. Perspectives on the engineering analogy. Dashed line indicates a mediation or influential effect.

\section{Conceptualisation of Gender Minority Stress from Social Theories}

The integrative conceptualisation of the GMSF that this paper proposes is not based on one congruous theory, but is rather informed by several sociological and psychological theoretical frameworks (Meyer, 1995, 2003). Below is the discussion of the frameworks that outline the role of institutionalised ideologies and social norms that consign TGD people to a minority position.

In Meyer's original conceptualisation of MST (2003), reference was made to Merton's (1968) work on institutionalised social norms in relation to minority groups. According to Merton, social norms generate stressors that conflict with the minority cultures, and with the interests of minority groups. In societies where distinctions of social status between dominant groups (e.g., cisgender people) and minority groups (e.g., TGD people) are particularly prominent, there is a tendency for dominant groups to marginalise minority groups. Meyer (1995) drew on Societal Reaction Theory to provide explanations for the "processes by which persons come to be defined as deviant" by societies (Kitsuse, 1962, p. 248). A behaviour or social norm is considered "deviant" if it departs from the conventional 
understanding of an appropriate behaviour or social norm (Kitsuse, 1962). This theory assists in identifying the members of a minority group who are defined by social norms as deviant, as well as determining how these members are thus treated.

Cisnormativity refers to the assumption that it is "normal" for one's gender identity to reflect the physical sex assigned at birth in the expected way, and that both sex and gender are only binary (Baril \& Trevenen, 2014). Cisnormativity as an ideology and the institutionalised social norm of being cisgender (a prejudice known as cisgenderism) explain why TGD identity is treated as deviant by contemporary societies. Cisnormativity is used to describe situations where people fail or refuse to comprehend the identities or experiences of TGD people (Riggs, Ansara, \& Treharne, 2015). This ideology privileges cisgender people, as it reinforces the understanding that there are only two valid genders (i.e., woman and man) and that these should always correlate with biological sex in the expected ways, delegitimising TGD people's own understanding of their genders and bodies (Ansara \& Hegarty, 2012; Baril \& Trevenen, 2014; Riggs et al., 2015). Cisnormativity is prejudical for TGD people, because it treats people as deviant if they decline, or are suspected of declining, the maintenance of a conventional consistency between genitalia and gender presentation (e.g., physical body and attire) as deviant. An example of pathologisation of TGD people is the usage of negative classifications in various versions of the Diagnostic Statistical Manual of Mental Disorders (DSM), such as gender identity disorder (American Psychiatric Association, 1994), which was more recently relabelled as gender dysphoria (American Psychiatric Association, 2013) but is still conceptualised as a divergence from norms requiring psychiatric attention. Examples like this demonstrate that the pathologisation of TGD people is embedded in the construction of gender within a cisnormative framework.

Cisnormativity also includes the misgendering of TGD people, which involves misclassifying TGD people based on dominant understandings of genders and bodies (Riggs 
et al., 2015). Misgendering of TGD people results in nonaffirmation, where surrounding people are unable or unwilling to acknowledge or use the appropriate name, pronoun or gender of a TGD person. For instance, a trans woman may be labelled as a man, addressed with male pronouns or referred to by a former male name. Those who identify as genderqueer or gender diverse may also be subjected to nonaffirmation, when surrounding people are unable to recognise them in a gender-neutral manner (Testa et al., 2015). Testa et al. (2015) identified nonaffirmation as a form of distal stressor in the GMSF framework.

Much psychological research is conducted from a cisnormative perspective (Ansara \& Hegarty, 2012). Psychological research has presumed being cisgender to be the social norm and most psychological researchers conceptualise human experience in strictly cisgender terms (Ansara \& Hegarty, 2012). Past psychological and medical researchers who have applied a cisnormative framework have at best neglected and invalidated the existence of TGD people. If psychological research assumes the population of interest to be cisgender, it is difficult to examine the issues affecting the TGD populations and to develop measures to address the health and social needs of TGD people. At its worst, psychological and psychiatric research has taken an active anti-trans stance, for example by attempting to produce evidence of the effectiveness of conversion therapies (Bernal \& Coolhart, 2012).

The Social Exclusion Framework provides explanations for the effects of marginalising social norms on members who are considered as deviant (Iwasaki et al., 2005). In this instance, cisgenderism causes TGD people to be at risk of social exclusion, as they face limited opportunities and access to resources at various levels. The U.S. National Transgender Discrimination Survey (NTDS) of 6,450 TGD people revealed that TGD people experience labour market exclusions (unemployment and under-employment); economic exclusions (poverty); institutional and medical exclusions (structural discrimination); social isolations (limited social networks); cultural exclusions (inability to live according to 
culturally accepted norms); and spatial exclusions (difficulty accessing public spaces and services) (Grant et al., 2011). The NTDS also identified the issue of accessing gender segregated bathrooms to be of concern among TGD people, especially for those who do not conform to reified expectations of how men and women should behave. A secondary analysis of NTDS data of 2,325 TGD college students on their experiences of accessing public bathroom found that 24.9\% had been denied access (Seelman, 2016). When correlated with frequency of suicide attempts, TGD college students who had been denied access to a school bathroom were more likely to have reported attempting suicides (Seelman, 2016).

\section{Conceptualisation of Gender Minority Stress from Social Psychological Theories}

Meyer (2003) also drew on social psychological theories in developing MST, in an attempt to uncover the social origins of negative societal reactions based on intergroup relations, developed through the process of categorisation. Identity categories are formed because there are distinctions between social groups (e.g., cisgender and TGD people). These categories serve as a platform for individuals within a group or multiple groups to self-define, thus forming a social identity of their own. Differences in social identities result in intergroup processes taking place between groups which are dominant or subordinate (Turner, 1999). In this instance, intergroup processes such as discrimination and prejudice constitute to minority stressors on the lives of TGD people.

In addition to the intergroup relations, Mead (1934) provided another perspective on the social origins of negative societal reactions, based on Symbolic Interaction Theory. This theory views social environments as providing people with meaning for their world and organisation of their experiences, positing that a person's sense of self develops through social interactions that ingrain meanings through symbols and signifiers. As part of growing up, a person observes and makes connections with the people surrounding them and their 
social environment, and learns to form judgements about social norms. In this instance, lack of acceptance of TGD identities and exposure to direct stereotypes and prejudice lead TGD people to realise the cisnormative nature of their social environment. As a result, TGD people may form negative self- regard in respect of their minority identity (Meyer, 2003). In particular, TGD people who hold negative self-regard, and experience internalised stigmatisation of their TGD identity may manifest high distress levels (Sánchez \& Vilain, 2009).

Meyer's shift from institutionalised social norms (e.g., being cisgender as the idealised norm) to a person's self-regard as the locus of stress, has been argued to be problematic based on interpretation of the engineering analogy (Riggs \& Treharne, 2017). The engineering analogy views stress in a social context, but in social psychological theories, stress is reduced to an individualised phenomenon. In these theories, stress is prioritised in relation to the subjective experiences of each TGD person towards negative societal reactions. Consequently, the importance of social norms in shaping how TGD people may be rendered legitimate targets of negative regard is diminished.

\section{Gender Minority Stressors}

Meyer $(1995,2003)$ made a distinction between distal sources of stress and proximal appraisals of stress, as in a continuum to classify the minority stressors that TGD people face into three processes - distal stressors, negative expectations, and internalised transphobia. While Riggs and Treharne (2017) made suggestions to reframe the language used to describe minority stress, they did not criticise Meyer's original intent to account for the effects of discrimination, nor the potential utility of MST if stressors are not considered to be purely resultant from personal internalisation of stigma.

\section{Distal stressors}


The first process relating to discrimination in MST is distal stressors, which are events experienced by people with minority identities, and range from everyday events of discrimination or microaggressions to other factors. Testa et al. (2015) posited that expressions of TGD identity expose TGD people to distal stressors that comprise genderbased victimization (verbal or physical acts committed against TGD people); gender-based rejection (rejection or non-acceptance by people, institutions, and communities); genderbased discrimination (difficulty accessing housing, employment, medical care, or legal documents) and non-affirmation (internal sense of gender identity of TGD people is not recognised by others). As purportedly objective factors, distal stressors are conceptualised as independent of personal identification and associated with the assigned minority position within a society (Meyer, 2003). For example, a woman who was assigned male at birth and has since undergone a medical gender transition so that her body is "female" might not selfidentify as trans. However, she may still be subjected to a similar degree of distal stressors as other trans women because she is perceived as a "trans" by others.

The U.S. Transgender Survey of 27,715 TGD people discovered that incidences of victimisation were relatively common among TGD people (James et al., 2016). Approximately half (48\%) of participants in this study reported experiencing at least one form of victimisation such as verbal harassment, physical attack and sexual assault. When compared to cisgender counterparts, the New Zealand Youth'12 study of 96 TGD and 8070 cisgender high school students, found a threefold increase in the percentage of TGD students who reported incidences of bullying on a weekly basis (17.6\% vs 5.8\%) (Clark et al., 2014). In the same study, TGD students were significantly more likely than their cisgender counterparts to report being physically harmed by another person (49.9\% vs $32.5 \%)$. These differences in experiences demonstrate some of the ways that TGD people are more likely to experience distal stressors than the cisgender population. 


\section{Negative expectations}

Based on the minority stressors continuum that Meyer $(1995 ; 2003)$ postulated, distal stressors play an important role in influencing a person's perceptions and appraisals of stress. He conceptualised the subjective appraisals of minority stress as proximal stressors, which comprise the second and third processes relating to discrimination in MST (Meyer, 2003). The second process is negative expectations for future events, which describes the anxiety of TGD people in anticipating distal stressors because of previous experiences with prejudice and discrimination toward their TGD identity (Hatzenbuehler \& Pachankis, 2016). Evidence of negative expectations for future events among TGD was observed in the New Zealand Youth'12 study, where more than half of TGD students $(53.5 \%)$ reported being afraid that someone at school would hurt or bother them (Clark et al., 2014). Comparatively, cisgender students (39.8\%) were less likely to report to be fear of victimisation (Clark et al., 2014). Proximal stressors also includes nondisclosure, which describes attempts made by TGD people to conceal their TGD identity in an attempt to protect themselves or others close to them from directly experiencing distal stressors (Testa et al., 2015). During this process, TGD people often feel the need to be vigilant in deciding if they should conceal or disclose their TGD identity. The maintenance of a vigilant state when anticipating discrimination can result in high levels of distress (Meyer, 1995).

\section{Internalised transphobia}

For TGD people, the third process relating to discrimination in MST is the proximal stressors of internalised transphobia or the internalisation of negative societal attitudes about one's own TGD identity and TGD people as a social group (Hatzenbuehler \& Pachankis, 2016). As a case in point, a study of 482 Argentinian TGD people found that more than half (55.8\%) of participants experienced some forms of internalised transphobia, such as feeling 
ashamed and low self-esteem in relation to their TGD identity (Marshall et al., 2016). Often, these responses arise from pervasive exposure to negative societal reactions resulting from cisnormativity (Marshall et al., 2016).

In contrast with the distal stressors, negative expectations for future events, nondisclosure and internalised transphobia are theorised in MST to be more subjective and rely on internal appraisals among minority people (Meyer, 2003; Testa et al., 2015). The degree of anticipation of stressful events and internalised negative attitudes, is thus, theorised to vary for each TGD person. Hatzenbuehler's Psychological Mediation Framework postulates that MST does not sufficiently explain the proximal pathways through which distal stressors are implicated in the process of contributing to mental health problems (Hatzenbuehler, 2009). This is because MST does not investigate the general psychological processes of people of minority groups, as part of the understandings of proximal stressors. In this instance, general psychological processes refers to the "common vulnerabilities in psychological and social processes" that are shared by both cisgender and TGD people (Hatzenbuehler, 2009, p. 712). The Psychological Mediation Framework suggests that distal stressors may cause TGD people to be more vulnerable to general psychological processes such as maladaptive emotion regulations, social exclusions, and feelings of hopelessness. However, this review focuses on minority stressors that are specific to TGD people and therefore characteristically different from stressors that apply to cisgender people.

In the understanding of proximal stressors experienced by TGD people, there is a need to recognise their linkages to cisnormativity. Allowing for these linkages is necessary to address the disjuncture between the social context and the individualised conceptualisation of internalisation. As suggested by Riggs and Treharne (2017), applications of the MST is often limited to a reified understanding of proximal stressors as a form of internalised cognition 
that enacts the theoretical switch from stressful social environments (as the results of marginalising social norms) to a personalised account.

\section{Gender Minority Protective Factors}

Despite not fitting into the dominant engineering analogy that views stress in a social context, Meyer's (2003) and Hatzenbuehler's (2009) position of speculating stress as proximal is important, as it opens up the possibility for protective factors (i.e., resilience and coping) to buffer against the negative ramification of minority stressors. Protective factors for TGD people can be divided into individual-level and social-level (Testa et al., 2015). Within this conceptualisation, there is one individual-level protective factor - identity pride, while social-level protective factors include community connectedness, family support, and culture connectedness.

Singh, Hays, and Watson (2011) reported the primary aspect of resilience among TGD people as identity pride, which is the ability to define one's own gender identity and embrace one's self-worth. Notably, embracing self-worth was a critical component of enabling a strong 'internal coach' to negotiate negative messages from societal discrimination and internalised transphobia (Singh et al., 2011, p. 23). TGD people who have a strong TGD identity have a reinforced sense of self, and are also more able to advocate for themselves in a cisnormative social environment (Singh et al., 2011).

TGD people who have been exposed to resources pertaining to social-level protective factors (e.g., peer networks and connectedness to trans-specific support groups) have reported these social supports as being beneficial for them to learn about medical resources, speak out about political concerns, and strengthen their TGD social networks (Singh et al., 2011). In particular, developing a sense of community connectedness with TGD-specific support groups and social networks was found to serve as a counterpoint to minority stressors by 
facilitating social-level coping resources, such as fostering connections with other TGD people, finding positive role models, and normalising emotional reactions related to discrimination through shared experiences with TGD peers (Pflum, Testa, Balsam, Goldblum, \& Bongar, 2015).

Differences in values across Western and non-Western contexts mean Western understandings of TGD experiences are inadequate for understanding TGD experiences in other cultural contexts; including many indigenous culture (Kirmayer, Sehdev, Whitley, Dandeneau, \& Isaac, 2009; Walters \& Simoni, 2002). Although there are important convergences between the proposed protective factors and indigenous concepts of health and wellbeing, there is a definite need to integrate indigenous perspectives to understand the specific minority stressors that indigenous TGD people face (Kirmayer et al., 2009; Walters \& Simoni, 2002). For instance, the indigenous people in Aotearoa/New Zealand known collectively as Māori recognise health more holistically, in comparison to the biomedical manner that is typical of Western views of health that has often neglected the ways in which health is interconnected and interdependent with other components of well-being, such as family support and cultural connectedness (Durie, 1999; Rochford, 2004).

Family members are often characterised as 'primary group members' with whom TGD people can form emotional ties, and are thus viewed as important or influential in their lives (Thoits, 2011). In the U.S. Transgender Survey, a comparison to TGD people with unsupportive family members found that TGD people who receive positive familial responses toward their TGD identity are less likely to experience psychological distress (31\% vs 50\%), as they are better equipped with coping mechanisms to deal with minority stressors (James et al., 2016). When family support is translated into the indigenous context, this factor also exerts a protective effect to buffer against the impacts of minority stressors (Pettingell et al., 2008). For example, a study of cisgender Alaskan indigenous young adults found that those 
who get along well with family members or have someone in their family who cares for their wellbeing, are protected against suicidality (Pettingell et al., 2008).

The GMSF fails to take into account cultural connectedness among TGD people, which is an important protective factor in mitigating the negative impacts of minority stressors (Scheim et al., 2013; Walters \& Simoni, 2002). The Canadian Trans PULSE study of 433 TGD people (including 32 who identified as indigenous) found that the indigenous TGD people perceived community and spiritual sources of support as vital, with $19.0 \%$ of them having approached cultural community leaders for mental health support (Scheim et al., 2013). Cultural connectedness can be assessed with the cultural factors in the Indigenist Stress-Coping Model (Walters \& Simoni, 2002) that includes identity attitude (the extent to which one internalises or externalises one's attitude toward their cultural or ethnic background) and enculturation (learning and identifying with one's cultural or ethnic background), spiritual methods of coping, and traditional health practices.

\section{Consideration of Multiple and Intersecting Identities}

Past utilisations of MST (Meyer, 1995, 2003) and the GMSF (Testa et al., 2015) have been critiqued around the usage of broad identity terms to effectively refer to one particular population (Meyer, 2010, 2015). For instance, the term "TGD” often connotes only Western, middle class, or urban TGD people. Applications of the GMSF that do not include indigenous, underprivileged, or rural TGD people can lead to homogenised and limited understandings of TGD people's experiences, and consequently only advance the interests of sub-sections of the TGD population who are already relatively privileged (Meyer, 2010, 2015; Parent, DeBlaere, \& Moradi, 2013). Hence, a comprehensive understanding of gender minority stress requires consideration of multiple identities.

\section{Additive Approaches to Minority Stressors}


An additive approach treats marginalised identities as independent of each other, and sees them as combining to shape the experiences of a person or a group who possess more than one marginalised identity (Parent et al., 2013). MST illustrates this approach by referring to having two minority identities (e.g., TGD and ethnic-minority) as double jeopardy, and three minority identities (e.g., TGD, ethnic-minority, and rural) as triple jeopardy (Meyer, 2010). Within MST, people who face double or triple jeopardy would have higher prevalences of mental health problems because of the greater risks of minority stress resulting from adding other minority identities to the TGD identity, when compared to people who have a single minority identity (Jaspal, 2015; Meyer, 2010). In terms of social-level protective factors, the added minority identity may also restrict the ways in which TGD people with two minority identities identify and affiliate with TGD groups (Meyer, 2015). For instance, TGD people from ethnic-minority groups may end up marginalised within predominantly Western TGD support networks (Singh, 2013). Lessening community connectedness may in turn amplify the negative impact of gender minority stressors on these TGD people. Double jeopardy for TGD people is demonstrated in the U.S. National College Health Assessment-II, which reported ethnic-minority TGD students may experience exacerbated amount of minority stress in relation to their double minority identities (Lytle, Blosnich, \& Kamen, 2016).

However, not every person with an additional minority identity experiences greater effects of minority stress. Meyer (2010) proposed that because ethnic-minority people commonly have prior exposure of racism, they may be resilient to the effect of minority stressors related to their other minority identity. This is suggested in an analysis of data from New Zealand Youth'07 and '12 studies, which found that ethnic-minority TGD people have better mental health outcomes than Western TGD people (Chiang et al., 2017). People of two minority identities have also been found to develop resilience in a more effective manner, by 
drawing upon social-level resources (e.g. community support, family values, and cultural beliefs) to buffer against the impact of minority stressors on mental health outcomes (Sanders \& Munford, 2015). For instance, indigenous Māori culture in Aotearoa/New Zealand provides specific resources (e.g., spiritual practices, and community gatherings) that can be drawn on to address the effects of minority stressors (Sanders \& Munford, 2015). Western people, on the other hand, may be more susceptible to minority stressors because they lack collectivist cultural resources to provide them with a stronger positive sense of identity (Sanders \& Munford, 2015).

\section{Intersectional Approaches to Minority Stressors}

Intersectionality acknowledges that a person may possess multiple identities that result in distinctive individual and collective experiences (Parent et al., 2013). Compared to an additive approach that treats identity categories as mutually exclusive variables, an intersectional approach contends that multiple identity categories construct novel experiences that are not necessarily divisible into their component identities (Parent et al., 2013). The focus of intersectionality is the result of a "fusing" of multiple identity categories (Choo \& Ferree, 2010; Parent et al., 2013). For example, Singh (2013) used an intersectional approach to examine the resilience experiences of ethnic-minority TGD youth. An African American trans man participant described his transgender culture and Black culture as inseparable, and that the minority stress he had experienced was distinctive. Singh (2013) also found that ethnic-minority TGD youth who valued the interconnectedness of their ethnicity and gender were more likely to develop a sense of empowerment and pride.

Intersectionality is used to explore how multiple and overlapping prejudices (e.g. racism and cisgenderism) shape the experiences of those with multiple marginalised identities (Crenshaw, 1991; Parent et al., 2013). Often, these social norms and prejudices contribute to 
the construction of relative privileging and marginalising systems that impact on a person and their wider social groups (Parent et al., 2013). The emphasis of an intersectional approach aligns with the understanding of engineering analogy of MST, which focuses on how ideologies and social norms serve to marginalise or privilege certain groups of people. Intersectionality was briefly discussed in Meyer's work (Meyer, 2010) but there has yet been extensive research on the application of intersectionality in relation to MST and the GMSF. Detailed reviews and meta-analyses will be possible after further research has been conducted into the GMSF. This further research will need to take into consideration the multiple and simultaneous effects of marginalising ideologies on TGD people, who may be oppressed along the multiple axes of inequality.

The selection of either an additive or an intersectional approach when conducting research with TGD people with multiple marginalised identity would depend on the theoretical approach adopted by the researcher. An additive approach can be applied in quantitative studies where identity categories (e.g., ethnicity and gender) are appraised as predictor variables in the equation of MST to ascertain their influences on criterion variable (e.g., mental health outcomes) (Parent et al., 2013). The effect of the predictor variables are tested through their main effects (e.g., the independent effect of ethnicity and gender). At the same time, quantitative studies can apply an intersectional approach by testing interactive effects (e.g., the interaction outcomes of ethnicity and gender). Qualitative methods can also be employed to examine the issues of intersectionality, with specific advantages in generating a nuanced understanding of the complexities and multiplicities of experiences (Parent et al., 2013). An intersectional approach resolves the "problem of addition" that results from the way in which an additive approach treats identity categories as mutually exclusive variables. Well-designed quantitative research can advance understanding of intersecting identities by testing research questions that attend to the needs of people with multiple identities, and the 
operationalisation of outcome variables that can reflect the unique experiences of these people (Parent et al., 2013). An example of quantitative research that utilised intersectionality is Jefferson, Neilands, and Sevelius (2013), which adapted scales about experiences of racism and cisgenderism to explore the combined effects of these experiences on ethnic-minority trans women, and found that combined discrimination related to the likelihood of depression.

\section{Conclusions and Summary of Future Directions}

There is a growing body of research that has employed MST (Meyer, 1995, 2003, 2015) to elucidate mental health disparities among minority groups. MST has provided fundamental scaffolding to describe the stress process experienced by members of minority groups, although the utilisation of this theory has largely been taken for granted, and ongoing critical evaluation and expansion is required. This paper attends to the critiques of Meyer's initial account of minority stress, which includes the definition of minority stress based on the engineering analogy (Riggs \& Treharne, 2017), the lack of emphasis on institutionalised ideologies and social norms (Riggs \& Treharne, 2017), inadequate explanations of general psychological outcomes (Hatzenbuehler, 2009), and the lack of consideration of the specific experiences resulting from having multiple and intersecting identities (Meyer, 2010, 2015).

This paper expands on the GMSF (Testa et al., 2015) to highlight specific minority stressors and protective factors for TGD people. By drawing on the sociological and social psychological theories that were used to conceptualise MST, this paper analyses these perspectives using the lens of cisnormativity. In this instance, a critical understanding of the ideology of cisnormativity is used in relation to the GMSF to provide explanations for the source of stressful social environments that affect TGD people. Cisnormativity also serves to describe how cisgender identities are constructed as the ideal social norm, and how they are 
privileged at the expense of TGD identities whilst also being constrained by narrow cisgender norms.

While distal and proximal minority stressors in relation to the sexual minority groups have been widely tested among TGD people, most TGD mental health studies (e.g., Marshall et al., 2016; McCarthy, Fisher, Irwin, Coleman, \& Pelster, 2014) have neglected TGDspecific minority stressors, such as non-affirmation and nondisclosure of gender identity. These two stressors were found to contribute substantially to the minority stress experiences of TGD people (Testa et al., 2015), and therefore should be included when considering minority stressors for TGD people. This paper has also demonstrated the relevance of two additional protective factors in relation to the original GMSF (Testa et al., 2015), which are family support and cultural connectedness. Although these two factors have not yet been tested in the GMSF as a whole framework, specific studies have suggested that these factors correlate with positive mental health outcomes among TGD people (Klein \& Golub, 2016; Scheim et al., 2013). Assessment of cultural connections should be conducted in a culturally sensitive manner and could benefit from the application of indigenous frameworks of health promotion, such as the New Zealand Te Pae Māhutonga model (Durie, 1999) or the Samoan Fa'afaletui model (Tamasese, Peteru, Waldegrave, \& Bush, 2005). Future TGD research should operationalise family support and cultural connectedness in ways that align with indigenous definitions of health, which often recognise health as interconnected and interdependent with other components of wellbeing (e.g., spirituality and connectedness to physical environments) (Kirmayer et al., 2009; Walters \& Simoni, 2002).

This paper has explored the intersection of ethnicity and gender, in relation to overlapping effects of racism and cisgenderism on TGD people's experiences of minority stressors and their mental health outcomes. The inclusion of an intersectional approach is important in TGD health research, in order to facilitate a shift in focus from the unitary effect 
of cisgenderism to the intersections among multiple systems of oppression. This intersectional shift would allow for a greater consideration of systemic inequalities within the TGD populations. There are other identities and systems of oppression that may affect the health outcomes of TGD people that we have not explored in this paper - these include those related to sexuality, religion, disability status, and socioeconomic status. Constraints in resources and knowledge within specific research projects may preclude a simultaneous analysis of every form of inequality, but studies that focus on TGD identities without at least considering other axes of identity are inadequate and reductive.

In conclusion, this paper argues that the GMSF is a useful framework for understanding TGD-specific minority stressors and protective factors, and their roles in predicting mental health outcomes. However, the original GMSF is not comprehensive enough. This paper proposes the integration of the GMSF into a broader culturally-embedded framework which would include indigenous perspectives and intersectional approaches, in order to adequately account for the gender minority stressors experienced by TGD people, whilst accounting for the importance of family support and culture connectedness.

\section{Conflict of Interest}

The authors declare that they have no conflict of interest.

ORCID ID: Kyle K.H. Tan 0000-0001-6831-7045 


\section{References}

Adams, N., Pearce, R., Veale, J., Radix, A., Castro, D., Sarkar, A., \& Thom, K. C. (2017). Guidance and ethical considerations for undertaking transgender health research and institutional review boards adjudicating this research. Transgender Health, 2(1), 165175. doi:10.1089/trgh.2017.0012

Alexeyeff, K., \& Besnier, N. (2014). Gender on the edge: Identities, politics, transformations. In N. Besnier \& K. Alexeyeff (Eds.), Gender on the edge: Transgender, gay, and other Pacific Islanders. Honolulu, HI: University of Hawai'i Press.

American Psychiatric Association. (1994). Diagnostic and statistical manual of mental disorders : DSM-IV. Washington, DC: American Psychiatric Association.

American Psychiatric Association. (2013). Diagnostic and statistical manual of mental disorders (5th ed.). Arlington, VA: American Psychiatric Publishing.

American Psychological Association. (2015). Guidelines for psychological practice with transgender and gender nonconforming people. American Psychologist, 70, 832-864. doi:10.1037/a0039906

Ansara, Y. G., \& Hegarty, P. (2012). Cisgenderism in psychology: Pathologising and misgendering children from 1999 to 2008. Psychology \& Sexuality, 3, 137-160. doi:10.1080/19419899.2011.576696

Baril, A., \& Trevenen, K. (2014). Exploring ableism and cisnormativity in the conceptualization of identity and sexuality 'disorders'. Annual Review of Critical Psychology, 11(1), 389-416.

Bernal, A. T., \& Coolhart, D. (2012). Treatment and ethical considerations with transgender children and youth in family therapy. Journal of Family Psychotherapy, 23, 287-303. doi:10.1080/08975353.2012.735594 
Chiang, S.-Y., Fleming, T., Lucassen, M., Fenaughty, J., Clark, T., \& Denny, S. (2017). Mental health status of double minority adolescents: Findings from national crosssectional health surveys. Journal of Immigrant and Minority Health, 19, 499-510. doi:10.1007/s10903-016-0530-z

Choo, H. Y., \& Ferree, M. M. (2010). Practicing intersectionality in sociological research: A critical analysis of inclusions, interactions, and institutions in the study of inequalities. Sociological Theory, 28, 129-149. doi:10.1111/j.1467-9558.2010.01370.x

Clark, T. C., Lucassen, M. F. G., Bullen, P., Denny, S. J., Fleming, T. M., Robinson, E. M., \& Rossen, F. V. (2014). The health and well-being of transgender high school students: Results from the New Zealand Adolescent Health Survey (Youth'12). Journal of Adolescent Health, 55(1), 93-99. doi:10.1016/j.jadohealth.2013.11.008

Clarke, V., Ellis, S. J., Peel, E. A., \& Riggs, D. W. (2010). Lesbian, gay, bisexual, trans and queer psychology: An introduction. Cambridge, United Kingdom: Cambridge University Press.

Crenshaw, K. (1991). Mapping the margins: Intersectionality, identity politics, and violence against women of color. Stanford Law Review, 43, 1241-1299. doi:10.2307/1229039

Dhejne, C., Van Vlerken, R., Heylens, G., \& Arcelus, J. (2016). Mental health and gender dysphoria: A review of the literature. International Review of Psychiatry, 28(1), 4457. doi:10.3109/09540261.2015.1115753

Durie, M. (1999). Te Pae Mahutonga: A model for Māori health promotion. Health Promotion Forum of New Zealand Newsletter 49.

Grant, J. M., Mottet, L. A., Tanis, J., Harrison, J., Herman, J. L., \& Keisling, M. (2011). Injustice at every turn: A report of the National Transgender Discrimination Survey. Retrieved from Washington, DC: National Center for Transgender Equality and National Gay and Lesbian Task Force 
Hatzenbuehler, M. L. (2009). How does sexual minority stigma "Get under the skin"? A psychological mediation framework. Psychological Bulletin, 135, 707-730. doi:10.1037/a0016441

Hatzenbuehler, M. L., \& Pachankis, J. E. (2016). Stigma and minority stress as social determinants of health among lesbian, gay, bisexual, and transgender youth: Research evidence and clinical implications. Pediatric Clinics of North America, 63, 985-997. doi:10.1016/j.pcl.2016.07.003

Iwasaki, Y., Bartlett, J., MacKay, K., Mactavish, J., \& Ristock, J. (2005). Social exclusion and resilience as frameworks of stress and coping among selected non-dominant groups. International Journal of Mental Health Promotion, 7, 4-17. doi:10.1080/14623730.2005.9721870

James, S. E., Herman, J. L., Rankin, S., Keisling, M., Mottet, L., \& Anafi, M. (2016). The report of the 2015 U.S. Transgender Survey. Retrieved from Washington, DC: National Center for Transgender Equality

Jaspal, R. (2015). Non-heterosexual British Asian men: Social representations, identity and social relations. In W. J.D. (Ed.), International encyclopedia of the social \& behavioral sciences (pp. 861-866). Oxford, Amsterdam: Elsevier.

Jefferson, K., Neilands, T. B., \& Sevelius, J. (2013). Transgender women of color: Discrimination and depression symptoms. Ethnicity and Inequalities in Health and Social Care, 6, 121-136. doi:10.1108/EIHSC-08-2013-0013

Kirmayer, L. J., Sehdev, M., Whitley, R., Dandeneau, S. F., \& Isaac, C. (2009). Community Resilience: Models, Metaphors and Measures. Journal of Aboriginal Health, 7(1), 62117.

Kitsuse, J. I. (1962). Societal reaction to deviant behavior: Problems of theory and method. Social Problems, 9, 247-256. doi:10.2307/799235 
Klein, A., \& Golub, S. A. (2016). Family rejection as a predictor of suicide attempts and substance misuse among transgender and gender nonconforming adults. $L G B T$ Health, 3, 193-199. doi:10.1089/lgbt.2015.0111

Koolhaas, J. M., Bartolomucci, A., Buwalda, B., de Boer, S. F., Flügge, G., Korte, S. M., . . . Fuchs, E. (2011). Stress revisited: A critical evaluation of the stress concept. Neuroscience \& Biobehavioral Reviews, 35, 1291-1301. doi:10.1016/j.neubiorev.2011.02.003

Lytle, M. C., Blosnich, J. R., \& Kamen, C. (2016). The association of multiple identities with self-directed violence and depression among transgender individuals. Suicide and Life-Threatening Behavior, 46, 535-544. doi:10.1111/sltb.12234

Marshall, B. D. L., Socías, M. E., Kerr, T., Zalazar, V., Sued, O., \& Arístegui, I. (2016). Prevalence and correlates of lifetime suicide attempts among transgender persons in Argentina. Journal of Homosexuality, 63, 955-967. doi:10.1080/00918369.2015.1117898

McCarthy, M. A., Fisher, C. M., Irwin, J. A., Coleman, J. D., \& Pelster, A. D. K. (2014). Using the Minority Stress Model to understand depression in lesbian, gay, bisexual, and transgender individuals in Nebraska. Journal of Gay \& Lesbian Mental Health, 18, 346-360. doi:10.1080/19359705.2014.908445

McNeil, J., Ellis, S. J., \& Eccles, F. J. R. (2017). Suicide in trans populations: A systematic review of prevalence and correlates. Psychology of Sexual Orientation and Gender Diversity, 4, 341-353. doi:10.1037/sgd0000235

Mead, G. H. (1934). Mind, Self, and Society. Chicago, CHI: University of Chicago Press. Merton, R. K. (1968). Social theory and social structure. New York, NY: Free Press. Meyer, I. H. (1995). Minority stress and mental health in gay men. Journal of Health and Social Behavior, 36(1), 38-56. doi:10.2307/2137286 
Meyer, I. H. (2003). Prejudice, social stress, and mental health in lesbian, gay, and bisexual populations: Conceptual issues and research evidence. Psychological Bulletin, 129, 674-697. doi:10.1037/0033-2909.129.5.674

Meyer, I. H. (2010). Identity, stress, and resilience in lesbians, gay men, and bisexuals of color. The Counseling Psychologist, 38, 442-454. doi:10.1177/0011000009351601

Meyer, I. H. (2015). Resilience in the study of minority stress and health of sexual and gender minorities. Psychology of Sexual Orientation and Gender Diversity, 2, 209-213. doi: $10.1037 /$ sgd0000132

Millet, N., Longworth, J., \& Arcelus, J. (2017). Prevalence of anxiety symptoms and disorders in the transgender population: A systematic review of the literature. The International Journal of Transgenderism, 18(1), 27-38. doi:10.1080/15532739.2016.1258353

Parent, M., DeBlaere, C., \& Moradi, B. (2013). Approaches to research on intersectionality: Perspectives on gender, LGBT, and racial/ethnic Identities. Sex Roles, 68, 639-645. doi:10.1007/s11199-013-0283-2

Pettingell, S. L., Bearinger, L. H., Skay, C. L., Resnick, M. D., Potthoff, S. J., \& Eichhorn, J. B. (2008). Protecting urban American Indian young people from suicide. American Journal of Health Behavior, 32, 465-476. doi:10.5993/AJHB.32.5.2

Pflum, S. R., Testa, R. J., Balsam, K. F., Goldblum, P. B., \& Bongar, B. (2015). Social support, trans community connectedness, and mental health symptoms among transgender and gender nonconforming adults. Psychology of Sexual Orientation and Gender Diversity, 2, 281-286. doi:10.1037/sgd0000122

Riggs, D. W., Ansara, G. Y., \& Treharne, G. J. (2015). An evidence-based model for understanding the mental health experiences of transgender Australians. Australian Psychologist, 50(1), 32-39. doi:10.1111/ap.12088 
Riggs, D. W., \& Treharne, G. J. (2017). Decompensation: A novel approach to accounting for stress arising from the effects of ideology and social norms. Journal of Homosexuality, 64, 592-605. doi:10.1080/00918369.2016.1194116

Rochford, T. (2004). Whare Tapa Wha: A Māori model of a unified theory of health. Journal of Primary Prevention, 25(1), 41-57. doi:10.1023/B:JOPP.0000039938.39574.9e

Sánchez, F. J., \& Vilain, E. (2009). Collective self-esteem as a coping resource for male-tofemale transsexuals. Journal of Counseling Psychology, 56(1), 202-209. doi:10.1037/a0014573

Sanders, J., \& Munford, R. (2015). The interaction between culture, resilience, risks and outcomes: A New Zealand study. In L. C. Theron, L. Liebenberg, \& M. Ungar (Eds.), Youth resilience and culture (pp. 81-92). New York, NY: Springer.

Seelman, K. L. (2016). Transgender adults' access to college bathrooms and housing and the relationship to suicidality. Journal of Homosexuality, 63, 1378-1399. doi:10.1080/00918369.2016.1157998

Scheim, A. I., Jackson, R., James, L., Sharp Dopler, T., Pyne, J., \& R. Bauer, G. (2013). Barriers to well-being for aboriginal gender-diverse people: Results from the Trans PULSE Project in Ontario, Canada. Ethnicity and Inequalities in Health and Social Care, 6, 108-120. doi:10.1108/EIHSC-08-2013-0010

Singh, A. A. (2013). Transgender youth of color and resilience: Negotiating oppression and finding support. Sex Roles, 68(11-12), 690-702. doi:10.1007/s11199-012-0149-z

Singh, A. A., Hays, D. G., \& Watson, L. S. (2011). Strength in the face of adversity: Resilience strategies of transgender individuals. Journal of Counseling and Development, 89(1), 20-27. doi:10.1002/j.1556-6678.2011.tb00057.x

Tamasese, K., Peteru, C., Waldegrave, C., \& Bush, A. (2005). Ole Taeao Afua, the new morning: A qualitative investigation into Samoan perspectives on mental health and 
culturally appropriate services. Australian \& New Zealand Journal of Psychiatry, 39, 300-309. doi:10.1080/j.1440-1614.2005.01572.x

Testa, R. J., Habarth, J., Peta, J., Balsam, K., \& Bockting, W. (2015). Development of the gender minority stress and resilience measure. Psychology of Sexual Orientation and Gender Diversity, 2(1), 65-77. doi:10.1037/sgd0000081

Thoits, P. A. (2011). Mechanisms linking social ties and support to physical and mental health. Journal of Health and Social Behavior, 52, 145-161. doi: $10.1177 / 0022146510395592$

Turner, J. C. (1999). Some current issues in research on social identity and self-categorization theories. In N. Ellemers, R. Spears, \& B. Doosje (Eds.), Social identity: Context, commitment, content Oxford, United Kingdom: Blackwell.

Walters, K. L., \& Simoni, J. M. (2002). Reconceptualizing native women's health: An "Indigenist" stress-coping model. American Journal of Public Health, 92, 520-524. doi:10.2105/AJPH.92.4.520 